\title{
Further refinement of the Corbelling Theory for the equilibrium analysis of corbelled domes
}

https://doi.org/10.1515/cls-2019-0003

Received Jul 07, 2018; accepted Jul 23, 2018

\begin{abstract}
The aim of this paper is to present a further development of the Corbelling Theory [1] for assessing the structural safety of "false domes" constructions like Trulli, and more generally for corbelled domes. In particular, it is well-known that a corbelled dome of a Trullo is a layered thick shell and that only the thin inner layer (candela) has a structural role. The proposed procedure extends the capabilities of the approach proposed in [2] to more general load conditions, including the infill. The effectiveness of the proposed approach is discussed through the analysis of a paradigmatic case study of a Trullo located in Alberobello. Since the geometry of the construction plays a fundamental role in the structural behavior, the starting point of the analysis was an accurate 3D-laser scanner survey of the dome. The obtained results give some interesting insights about the role of the infill load on the equilibrium of such kind of corbelled domes.
\end{abstract}

Keywords: Corbelled vaults, Historic constructions, Equilibrium analysis, Corbelling theory

\section{Introduction}

Dry-stone corbelled domes are a paradigm for the vernacular architecture of all the Mediterranean area. Cabane in Provence, Mitato in Greece, Girna in Malta, Trulli in Italy, or the earthen-corbelled domes in the Aleppo region are just some remarkable examples.

From the architectural point of view, corbelled domes can be considered as the natural three-dimensional exten-

\footnotetext{
*Corresponding Author: Aguinaldo Fraddosio: Department of Civil Engineering and Architecture, Polytechnic University of Bari, Italy; Email: aguinaldo.fraddosio@poliba.it

Nicola Lepore: Department of Civil Engineering and Architecture, Polytechnic University of Bari, Italy; Email: nicola.lepore@poliba.it Mario Daniele Piccioni: Department of Civil Engineering and Architecture, Polytechnic University of Bari, Italy; Email: mariodaniele.piccionipoliba.it
}

sion of the concept of false arch: indeed, both the constructions are composed by a superposition of purely horizontal stone layers slightly cantilevered toward the center until meeting at the top [3]. Whereas at first sight, this kind of constructions may appear to be the result of a self-building process, actually they are the expression of a considerable constructive knowledge, handed down over time. In particular, the above architectural typology comes from a long historical process: corbelled domes were well-known since the Bronze Age, and several examples can be found in archeological sites in Italy (Tuscany) and in Greece as the renowned case of the Mycenaean Tholoi (XIV century B.C.).

In spite of their extensive presence, this kind of masonry constructions have received a scarce interest from the scientific community, and consequently, their mechanics is not completely understood yet. Indeed, the structural behavior of corbelled domes was studied in only a few works in the scientific literature, and this issue is not covered neither by traditional calculation techniques $[4,5]$, nor by standard limit equilibrium analysis applied to masonry structures [6-8]. Finally, also advanced structural analysis numerical approaches [9] appear to be uncapable of describing the peculiar mechanical behavior of corbelled domes.

The first studies expressly dedicated to the structural analysis of corbelled constructions were mainly focused on the analysis of Mycenaean Tholoi, in the assumption that the so-called "false dome" was behaving basically in the same way of a "false arch", where the latter is a construction made up of horizontal blocks simply superimposed and placed so that each block protrudes with respect to the underlying one. In this vein, a corbelling dome is considered as composed by a series of independent meridian false-arches, and it is assumed that forces can be transferred between two consecutive rings of stones only through vertical load paths. This approach is known as the Corbelling Theory (CT) and it was first proposed in [10] for the case of Mycenaean Tholoi. In the latter work, the analysis is performed by imposing the overturning equilibrium for each group of blocks composing the generic infinitesimal wedge of the dome. 
In [1] the CT was subsequently formalized in a rigorous treatment based on suitable equilibrium differential equations; within this theoretical framework the study of falsearches, tholoi, and Trulli is proposed.

The limitations of the CT are discussed in [11, 12] with reference to the well-known tholos "Treasury of Atreus" (IV century BC). By considering the masses involved in the equilibrium of the construction, i.e., the self-weight of each block applied in its centroid, the stabilizing and overturning moments and the ratio between them are evaluated. This calculation shows a meridian wedge wouldn't be able to sustain itself autonomously. This apparent paradox is due to the inadequacy of the standard CT in describing the structural behavior of corbelling domes.

Indeed, for corbelling domes, the spatial shape based on horizontal rings of stones with closed geometry gives a crucial contribution to the stability of the single meridian wedge. In particular, the accurate brickwork of the horizontal rings of stones, thanks to the high technical expertise in inserting by the force small pieces of stones between the vertical stone joints, makes them capable to bear compression forces along parallels. In this way, the overturning of a part of the meridian wedge is prevented by the blocks of the adjacent meridian segments: this actually determine a fully 3D shell behavior of the structures.

Notice that the capacity of transmitting compressive forces along parallels is yet available also when the construction of the rings is not completed, thanks to the friction between the lateral surfaces of adjacent courses. A practical evidence of this, and thus of the effectiveness of the described constructive technique, is provided by several examples of partially collapsed domes, showing that a finite-dimension wedge, even with a partial circumferential development, is still able to stand without the collaboration of the collapsed surrounded structure (Figure 1). Of course, the above described three-dimensional structural behavior is out of the capability of the standard CT.

In order to overcome this limitation, Rovero and Tonietti in [13] proposed a Modified Corbelling Theory (MCT) based on the balance of the stabilizing and the overturning moments imposed on a finite-dimension meridian wedge. This allows for taking in account the three-dimensional behavior of corbelling domes, based on the possibility of the transmission of forces along horizontal rings.

Recently, a further development of the approach in [13] has been proposed in [2], where a new integral formulation of the equilibrium problem for corbelling domes has been developed. We will call this approach from now on New Formulation of the MCT (NFMCT). The obtained results show the capability of the proposed theory of obtaining more accurate predictions of the actual behavior of

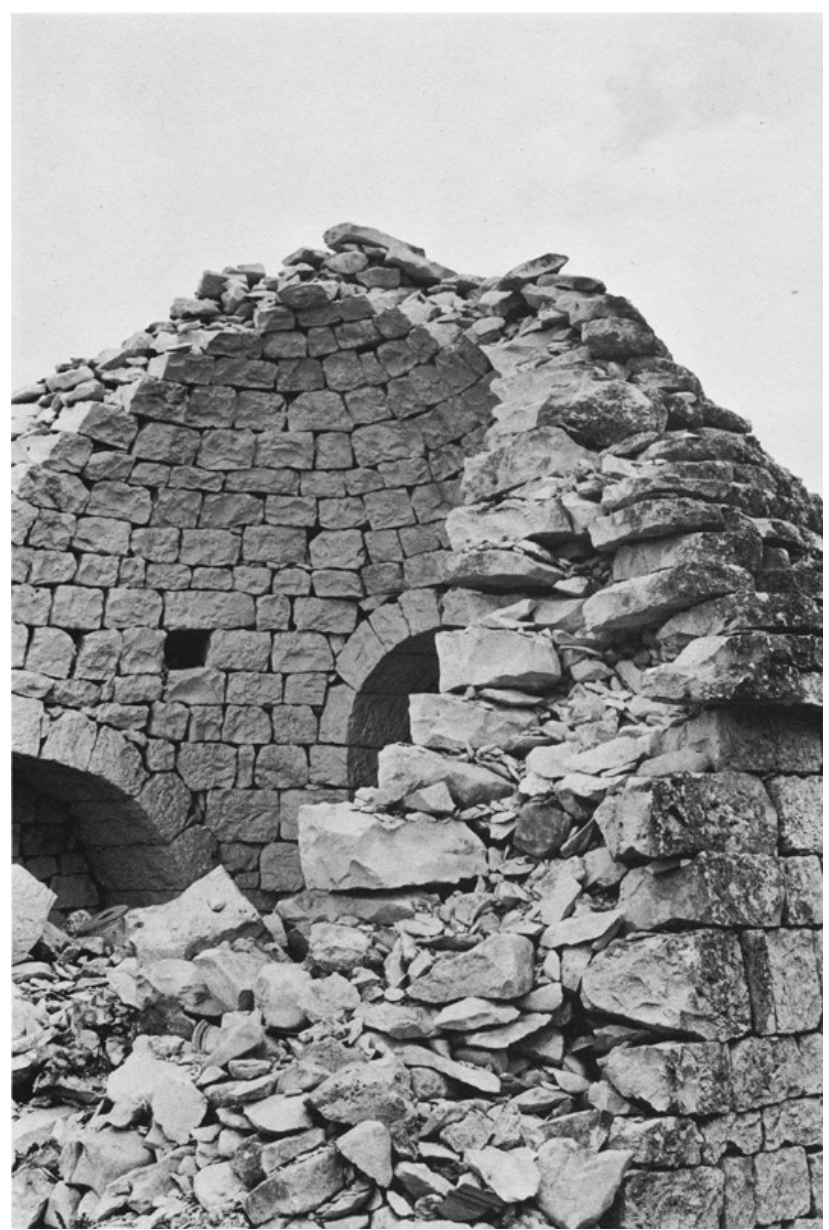

Figure 1: Example of the partial collapse of a Trullo vault [14].

the corbelling domes with respect to previous approaches in literature. Moreover, in [2] also a computational procedure based on the Thrust Network Analysis (TNA) $[15,16]$ is applied for understanding the structural behavior of corbelling domes.

For the sake of the completeness of the literature review we notice that also studies based on the application of the Finite Element Method have been proposed [17-19].

In this paper we start in Section 2 from the analysis of the building techniques and of the materials used for the construction of Trulli located in Alberobello, the wellknown city of Apulia (Italy), UNESCO site since 1996. Then, in Section 3 we underline the fundamental mechanical issues arising in the structural analysis of such kind of constructions, and we review the literature on this subject starting from the standard CT introduced in [1], passing from the MCT introduced in [13] and finally arriving to our improvement of the MCT, the NFMCT proposed in [2].

On this basis, we propose in Section 4 a further development of the analytical approach in [2], by taking into ac- 

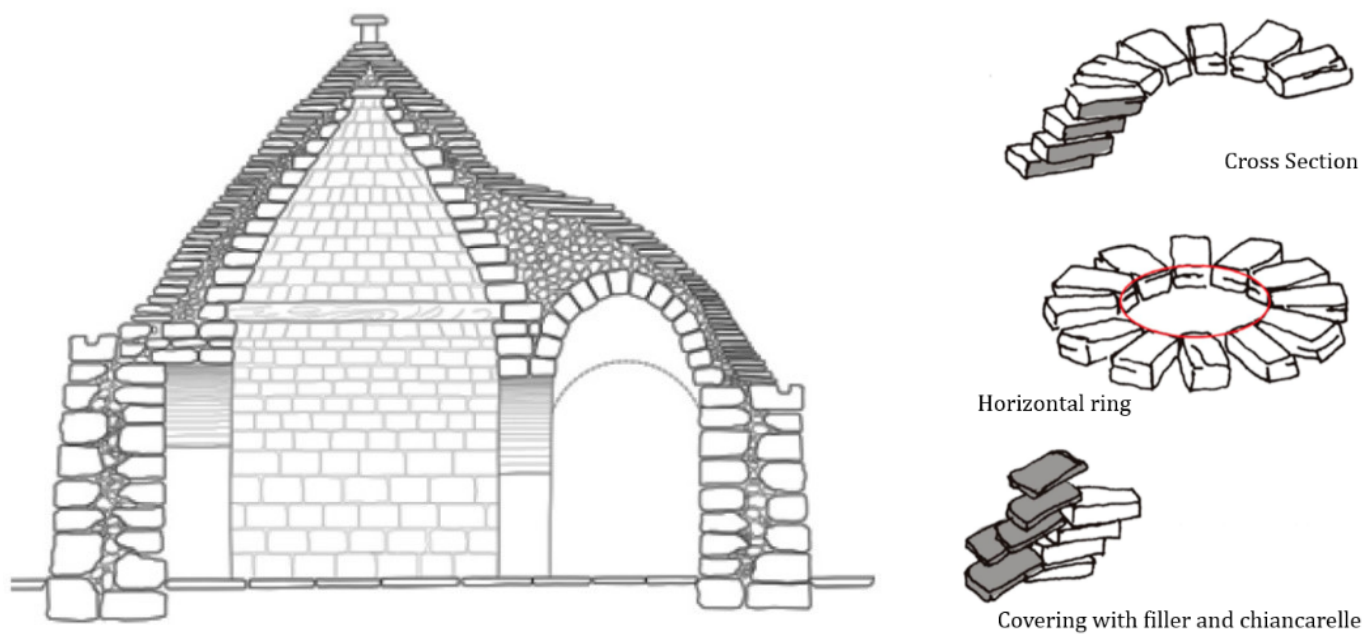

Figure 2: Constructive section and detail of an Italian Trullo [21].

count both the peculiar three-dimensional structural behavior of corbelled domes and the role of the infill load in the equilibrium of these structures, not yet considered in the literature. The aim of this refinement is that of obtaining a more accurate procedure for the static assessment of corbelled domes.

The effectiveness of the proposed approach is discussed in Section 5 through the analysis of a case study represented by a Trullo in Alberobello. Since the geometry of the construction plays a fundamental role in the structural response, our analysis is based on an accurate 3D-laser scanner survey of the dome. The obtained results show that our theory is capable of accurately describing the equilibrium of the examined masonry structures, and give some interesting insight about the role of the infill and the shape of the construction.

\section{Building technique of Trulli}

Trullo is typically a corbelled dry-stone building composed of two main elements: the basement and the pseudoconical dome [20].

The basement is commonly about $2 m$ high and can be circular o quadrangular. It is generally composed of two external walls made of large calcareous stones, whit the space between the two walls filled by rubble and waste [21]. The thickness of the basement is considerable since it generally ranges from $1 \mathrm{~m}$ to $2.5 \mathrm{~m}$.

The corbelled dome usually has a pseudo-conical shape and it is composed of three main shell layers (Figure 2). The inner layer, usually with a constant thickness, is called "candela" and it is built by overlapping layers of horizontal rings of stones. Each ring is made up of carefully chosen stone blocks, similar in form and size for each course, and arranged in concentric circles with decreasing diameters upwards until the last ring topped by a large stone called "chianca" or "serraglia" [21]. Special care is dedicated to achieving the continuity of the rings brickwork, by filling by force the gaps between blocks through little pieces of stones; this way, practically a rigid annular system is formed.

The outer shell layer is made by thin limestone slabs called "chiancarelle" that are put on the intermediate shell layer, made of filling material. Chiancarelle are inclined towards the outside in order to facilitate the outflow of meteoric water.

Only the inner layer has a structural role and, subsequently, the other two layers can be considered as nonstructural masses for the purpose of the structural analysis.

\section{Corbelling Theory: from the classic Benvenuto and Corradi approach to further developments}

The first rational theory formulated for understanding the mechanics of masonry corbelled domes - that, as described in Section 2, are characterized by a building technique quite different from conventional masonry domes is the mechanical model proposed by Benvenuto and Cor- 
radi [1], called Corbelling Theory (CT). The principal assumption of the CT approach is than that the mechanical behavior of a corbelled dome can be described as that of a polar series of corbelled arches. For this reason, each infinitesimal meridian slice of the dome should be capable to sustain itself independently to other meridian slices. Moreover, it is assumed that:

i rigid blocks;

ii perfectly horizontal layers;

iii no mortar and infinitesimal distance between overlapping layers;

iv prevented sliding between blocks;

The CT model is essentially based on the overturning limit equilibrium of an arbitrary infinitesimal meridian wedge of the corbelled vault. Since forces can be transferred only along the vertical direction, for each horizontal section of the wedge the limit equilibrium between the overturning and the stabilizing moment due to the weight of the part of the dome above the section is imposed.

The intrados and extrados profiles of the dome are expressed by continuous and smooth functions of the span length: then, by imposing the overturning limit equilibrium for each point of the intrados profile, the problem is formulated in differential form. In conclusion, CT leads to a differential problem in which the unknown functions are those describing the intrados profile and the extrados profile of the dome, respectively.

In [1], by introducing some approximations concerning the expression of the stabilizing moment, closed-form solutions of the problem are obtained.

A significant improvement of the CT is proposed in [13] by Rovero and Tonietti. The Authors first observe that the classic CT leads to a significant deviation between theoretical and actual profiles of corbelled domes; indeed, it is observed that the standard CT disregards the fully threedimensional shell behavior of corbelled domes. The latter, conversely, it is crucial for the equilibrium of the particular class of masonry constructions under investigation. In order to overcome this drawback, in [13] a Modified Corbelling Theory (MCT) it is proposed. This approach is derived from that in [1], but it is capable to taking into account also the horizontal interaction between infinitesimal meridian slices due to the transmission of forces along horizontal rings, allowed by the interlocking and the friction between adjacent blocks.

In particular, in order to describe the threedimensional shell behavior of corbelled domes, in [13] meridian slices with finite width are considered. The size of those slices depends on a parameter $\varphi$ representing the width angle of the meridian slice. Besides this, the other assumptions of the theory in [1] and, in particular, the same approximation in the expression of the stabilizing moment, are preserved.

By some representative examples, in [13] it is shown that MCT is capable of better results with respect to the classic $\mathrm{CT}$, although the more complex mathematical formulation renders the differential equilibrium problem essentially only numerically solvable.

It is worth to note that in [13] the value of $\varphi$ corresponding to the optimal solution is mechanically interpreted as the minimum width of a dome wedge that is capable to sustain itself independently from the rest of the dome.

Another step ahead towards a better understanding of the mechanics of corbelled domes is represented by the approach proposed in [2]. Starting from the observation that also the theoretical profiles predicted by MCT present a not negligible deviation from the actual profiles of corbelled domes, the Authors of [2] try to reach more accurate predictions by removing some relevant approximations in the expression of the stabilizing moment. The result is the New Formulation of the MCT (NFMCT), that further improve the MCT in terms of closeness between theoretical and actual profiles of a corbelled dome. Moreover, for NFMCT the best-fit solution is obtained for smaller angle $\varphi$ than those determined by the MCT, thus producing less conservative results. In the framework of the lower bound theorem of Limit Analysis this is another evidence of the improvements of the NFMCT on the MCT.

In Figure 3 the results obtainable by CT, MCT and NFMCT are compared for the same corbelled dome. In particular, since the solutions obtained by MCT and NFMCT depend on the angle $\varphi$, whereas the solutions obtained by CT do not depend on $\varphi$, in Figure 3 a a comparison between CT, MCT and NFMCT solutions (the latter two are obtained for $\varphi=50^{\circ}$ ) is performed. Notice that the theoretical profile predicted by CT is very different from the actual profile. An improvement is obtained by MCT, but the theoretical profile still significantly differs from the actual one. Finally, NFMCT solution is much closer to the actual profile. Figure $3 \mathrm{~b}$ compare the results obtained by MCT and NFMCT for the angles allowing the best fitting between theoretical and actual profiles. It is clear that NFMCT theoretical profile is practically coincident with the actual profile, whereas the MCT theoretical profile is still quite different from the actual one. 

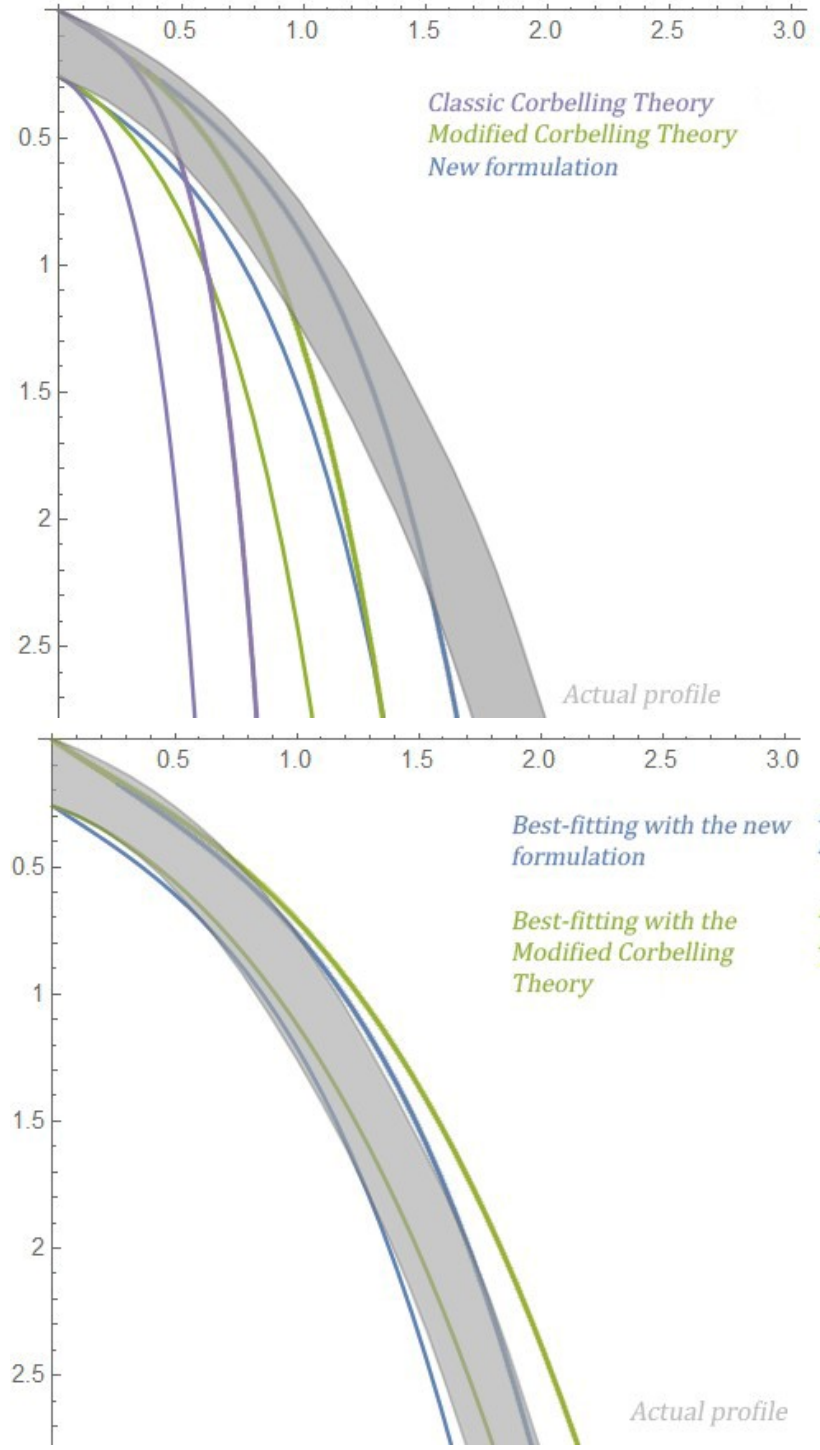

Figure 3: Comparison between the classic Corbelling Theory (CT), the Modified Corbelling Theory (MCT) and the new formulation of the Modified Corbelling Theory (NFMCT): (a) MCT and NFMCT bestfitting solutions; (b) CT, MCT and NFMCT solutions (the latter two are obtained for $\varphi=50^{\circ}$ ) [2].

\section{Further development of the NFMTC: the role of the infill load}

In the above-mentioned papers, the overturning limit equilibrium is always carried by considering as the only load the self-weight of the dome. Actually, the Trullo dome is a layered shell in which the thickness of the structural layer (candela) is often noticeably smaller than the overall thickness of the dome.

For this reason, here we propose a further improvement of the NFMCT in [2] by developing an approach ca- pable of taking into account also the role of the infill load in the limit equilibrium of the structure.

To this aim, the external surface of the dome must be identified. We observe that this surface is easily obtainable from a geometrical survey; anyway, such surface is very often pseudo-conical for the case of Trulli and so, the analytical expression of its profile in a cross section can be approximated to a straight line.

Now let us consider a finite dimension meridian wedge of the Trullo dome, whose angular width is represented by the angle $\varphi$, and whose plan and middle cross section are represented in Figure 4. For any point $P(x)$ belonging to the intrados curve $y(x)$ of the candela, it is possible to define, in the same way as in [2], the following two region of the candela: $P Q R$ and $P R A B$, whose self-weight contributes to the stabilizing moment $\left(M_{S}^{c}\right)$ and to the overturning moment $\left(M_{R}^{c}\right)$ for the equilibrium of the candela, respectively.
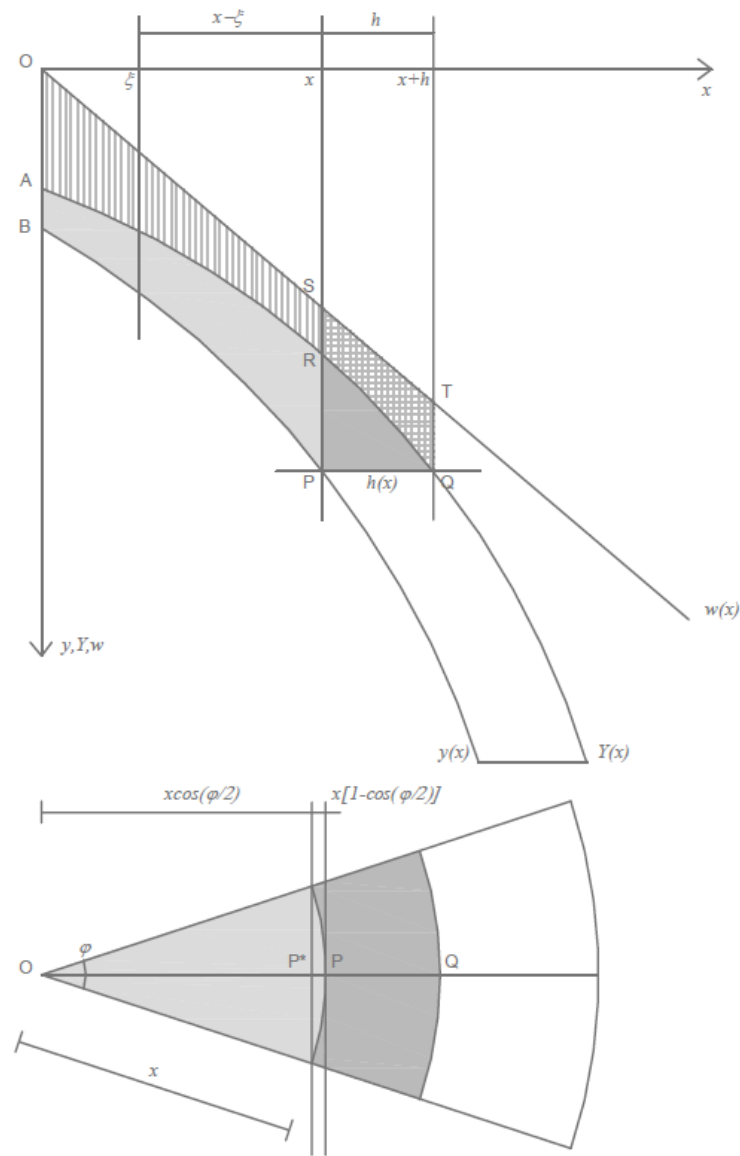

Figure 4: Finite dimension meridian wedge in plan and section, contributions to the stabilizing moment and the overturning moment of the self-weight and infill. 
Also in this case, due to the finite-size $\varphi$ of the meridian wedge, the position of the overturning axis moves toward the center of the dome, and intersect the middle cross section in a point $P^{*}$ instead of the point $\mathrm{P}$ belonging to the candela. Thus, the stabilizing moment $M_{S}^{c}$ and the overturning moment $M_{R}^{c}$ may be evaluated as follows:

$$
\begin{gathered}
M_{R}^{c}=\gamma_{c} \int_{0}^{x}(y(\xi)-Y(\xi))\left(x \cos \frac{\varphi}{2}-\xi\right)(\xi \varphi) d \xi, \\
M_{s}^{c}=\gamma_{c} \int_{x}^{x+h}(y(x)-Y(\xi))\left(\xi-x \cos \frac{\varphi}{2}\right)(\xi \varphi) d \xi,
\end{gathered}
$$

where $y(x)$ is the ordinate of the point $P$ on the intrados of the inner layer; $\gamma_{c}$ is the specific weight of the candela, and $h(x)$ is the horizontal thickness of the candela at $x$.

Called $w(x)$ the function defining the external profile of the dome in the middle cross section, with reference to Figure 4 it is possible to define other two regions: $R Q S T$ and $R S O A$. The latter are related to the contributions $M_{S}^{i}$ to stabilizing moment and $M_{R}^{i}$ to the overturning moment of the self-weight of the non-structural masses that lie above the extrados of the candela, respectively. Those contributions represent the effects of the infill on the equilibrium of the corbelled dome. In order to evaluate $M_{S}^{i}$ and $M_{R}^{i}$, let us consider again the rotation axis passing through the point $P^{*}$; it follows:

$$
\begin{aligned}
& M_{R}^{i}=\gamma_{i} \int_{0}^{x}(Y(\xi)-w(\xi))\left(x \cos \frac{\varphi}{2}-\xi\right)(\xi \varphi) d \xi, \\
& M_{S}^{i}=\gamma_{i} \int_{x}^{x+h}(Y(\xi)-w(\xi))\left(\xi-x \cos \frac{\varphi}{2}\right)(\xi \varphi) d \xi,
\end{aligned}
$$

where $\gamma_{i}$ is the homogenized specific weight of the nonstructural masses lying above the extrados of the candela (filler and chiancarelle); in particular, $\gamma_{i}$ may be evaluated as the weighted average of the specific weights of the filler and of the chiancarelle. Usually, it results $\gamma_{i}<\gamma_{c}$, but the value of $\gamma_{i}$ is not so much smaller than that of $\gamma_{c}$. For this reason and for the sake of the simplification of the calculations, in what follows we assume that $\gamma_{i}=\gamma_{c}$. Notice that this assumption is in favour of safety, since it leads to an overestimation of the contribution by the infill, which - as we will observe below - produces an instabilizing effect.

For the safety assessment of the corbelled dome we have to verify that

$$
M_{S} \leq \rho M_{R},
$$

where

$$
M_{S}=M_{S}^{c}+M_{s}^{i} ; M_{R}=M_{R}^{c}+M_{R}^{i},
$$

and $\rho \geq 1$ is a suitable safety coefficient.

The limit overturning equilibrium condition, studied in the following, is obtained from (5) by considering $\rho=1$.

For Trullo dome is reasonable to assume that the horizontal thickness is constant: $h(x)=h_{0}$; in this hypothesis, by substituting equations (1-4) in (5) the limit overturning equilibrium condition takes the form:

$$
\begin{aligned}
& \int_{0}^{x}(y(\xi)-Y(\xi))\left(x \cos \frac{\varphi}{2}-\xi\right)(\xi \varphi) d \xi \\
& +\int_{0}^{x}(Y(\xi)-w(\xi))\left(x \cos \frac{\varphi}{2}-\xi\right)(\xi \varphi) d \xi \\
& -\left[\int_{x}^{x+h}(y(x)-Y(\xi))\left(\xi-x \cos \frac{\varphi}{2}\right)(\xi \varphi) d \xi\right. \\
& \left.+\int_{x}^{x+h}(Y(\xi)-w(\xi))\left(\xi-x \cos \frac{\varphi}{2}\right)(\xi \varphi) d \xi\right]=0 .
\end{aligned}
$$

By following the same procedure in [2], we differentiate two times (7) with respect to $x$; this way, it is possible to reduce equation (7) to the following differential form:

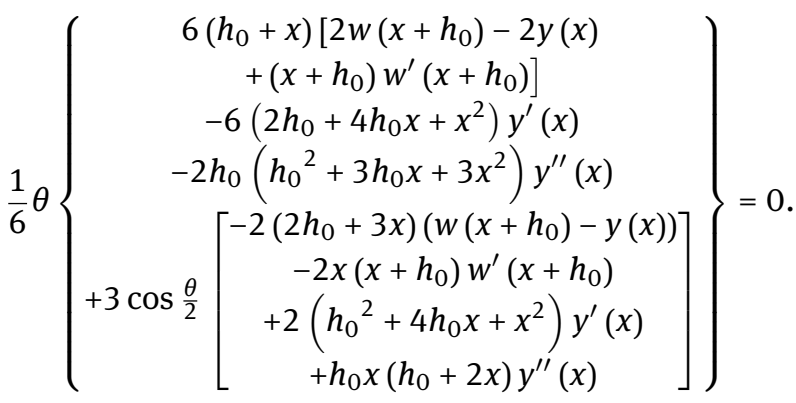

where, as it is assumed in $[1,2,13]$, the thickness $h(x)$ can be approximated as follows:

$$
h(x)=\frac{y(x)-Y(x)}{Y^{\prime}(x)} .
$$

In order to solve the differential problem above stated, we impose the following boundary condition for $x=0$, i.e., at keystone of the dome:

$$
Y(0)=k ; y(0)=k+m ; y^{\prime}(0)=n,
$$

where $k$ is the thickness of the infill at the crown while $m$ and $n$ depend on the geometry of the dome, $m$ is the vertical thickness of the candela at the crown and $n$ is the slope of the intrados curve at the crown. 
In the assumption that $w(x)$ is known, the system of differential equations (8)-(9) with the boundary conditions (10) can be numerically solved for finding, as the solutions, the function defining the "theoretical" profiles $y(x)$ and $Y(x)$ of the intrados and the extrados of the candela, respectively, that is, the profiles of the candela corresponding to the fulfillment of the overturning limit equilibrium.

Notice that the solution depends on the parameter $\varphi$, described in Section 4, that have to be fixed in advance. By varying the value of $\varphi$, and by solving iteratively the overturning equilibrium differential problem it is possible to identify the optimal value of $\varphi$, that is the value of $\varphi$ corresponding to the theoretical intrados and extrados profile of the candela that better approximate the actual profile of this structural layer of the corbelled dome (best-fit solution).

\section{Case study}

In order to discuss the proposed corbelling theory, that takes in to account also the role of the infill load in the equilibrium of a corbelled dome, our approach has been applied to a representative case study of a Trullo located in Alberobello (Figure 5).

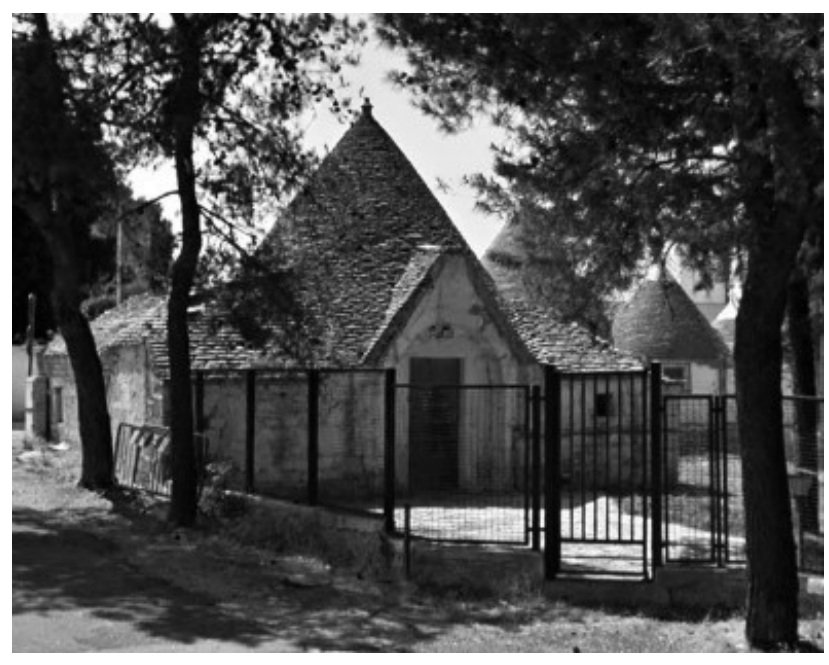

Figure 5: The case study: a Trullo in Alberobello.

The examined construction is a complex Trullo (Figure 6) composed by a central volume, covered by a corbelled dome, and several smaller added rooms covered by vaults of different types.
The basement is made by a thick-layered wall composed of two external layers of squared blocks of limestone that are filled with small stones and waste material. The blocks have different dimensions due to the necessity of obtaining a brickwork as regular as possible. The thickness of the basement varies from $0.8 \mathrm{~m}$ to $1.5 \mathrm{~m}$ (Figure 6).
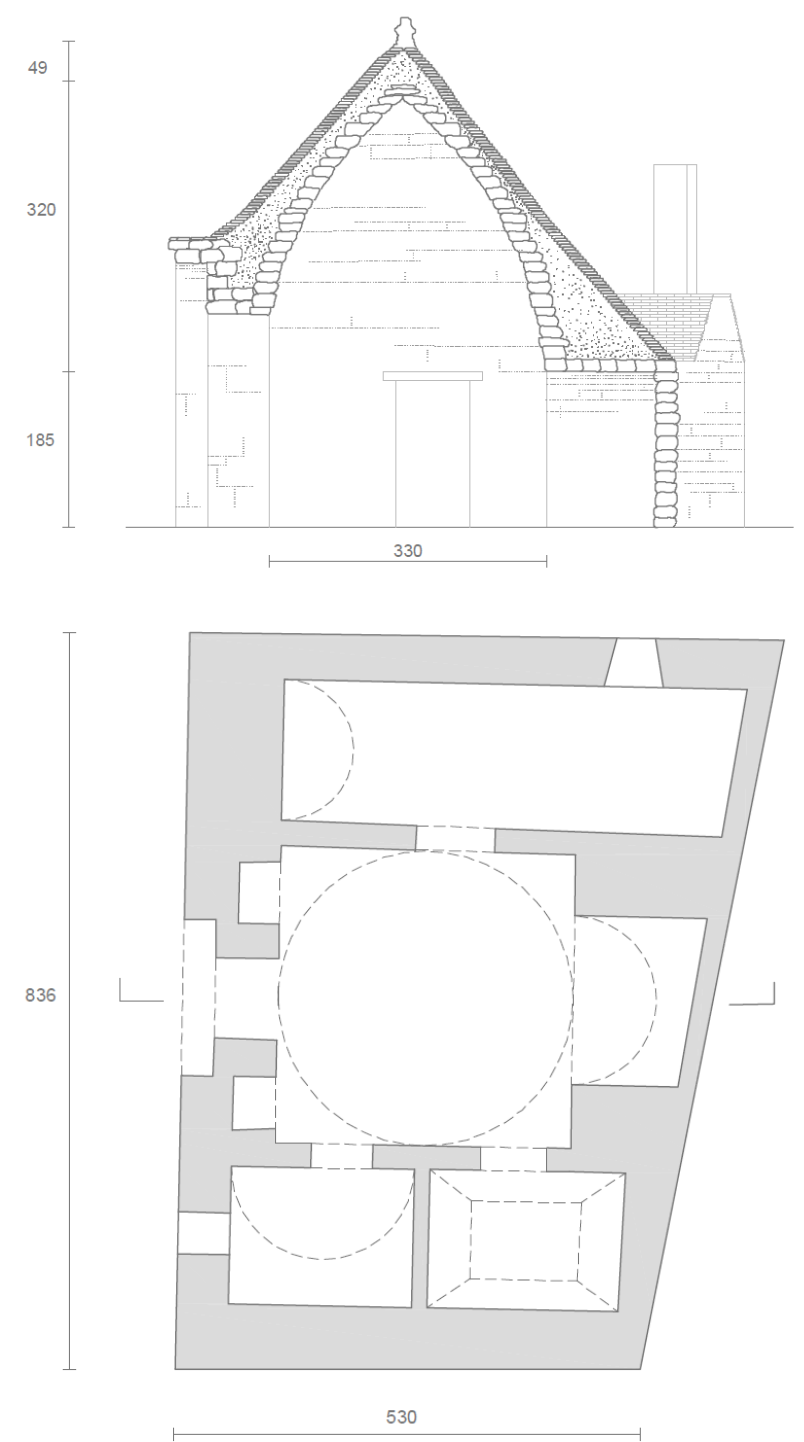

Figure 6: Cross-section and plan of the studied Trullo in Alberobello.

The performed analysis has been focused on the structural assessment of the central pseudo-conical corbelled dome of the Trullo. It covers a span of $3.30 \mathrm{~m}$ and the thickness of the structural layer (candela) could be considered constant and equal to $0.28 \mathrm{~m}$. 

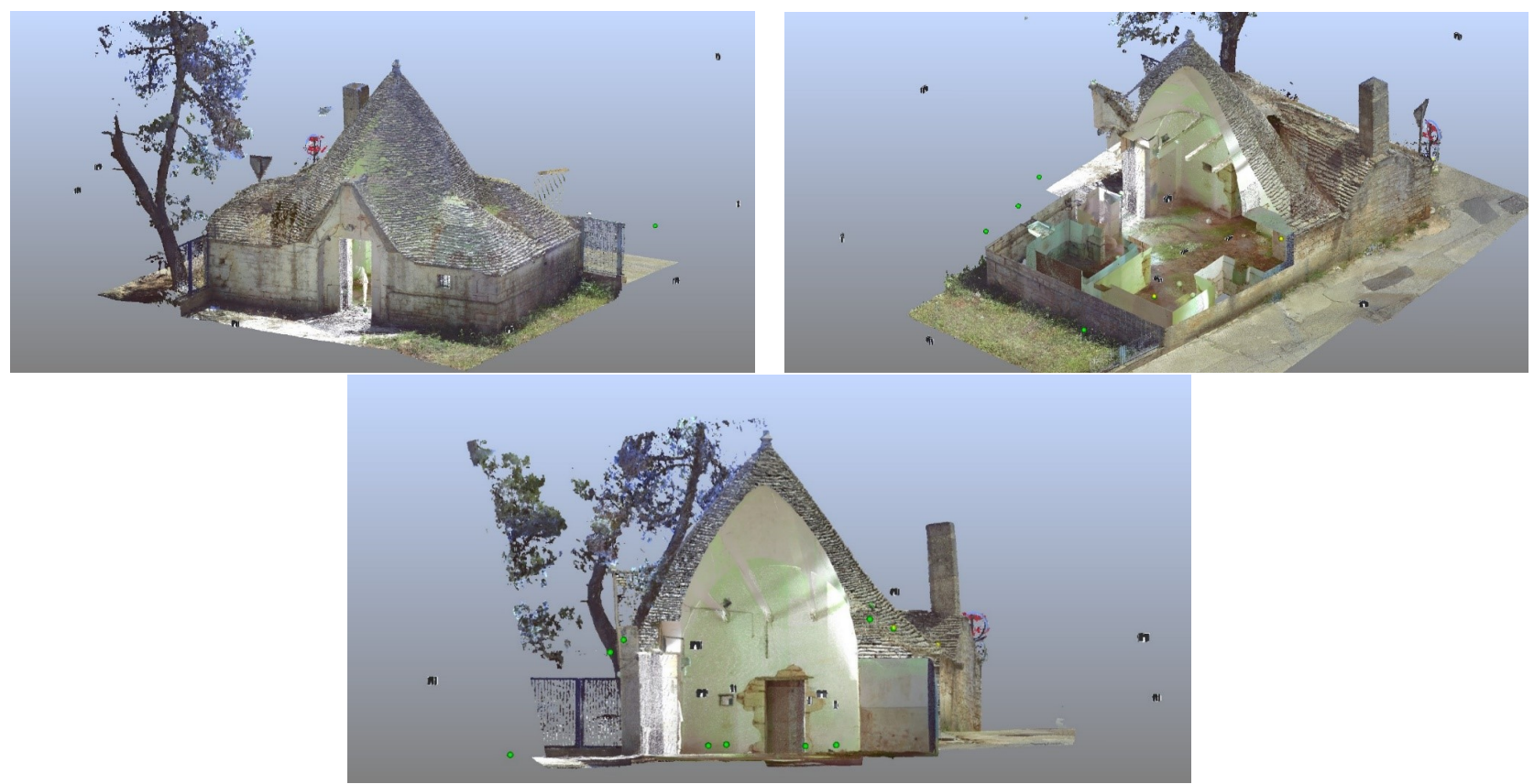

Figure 7: The cloud of points obtained from the 3D-laser scanner survey.

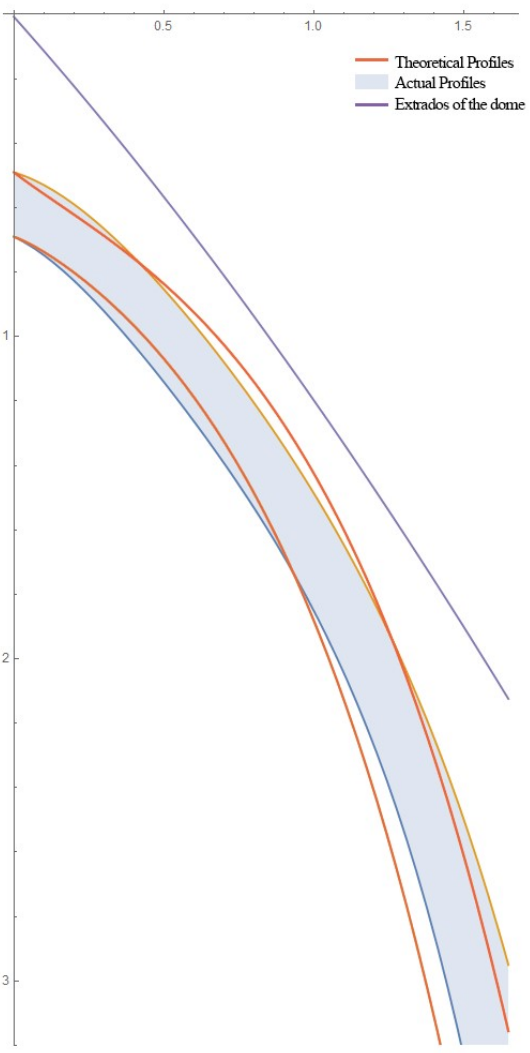

Figure 8: Actual and theoretical profiles of the candela obtained for $\varphi_{0}=41.27^{\circ}$ by NFMCT (that is, considering only the self-weight of the candela).
Since the strict connection between the shape and the structural behavior of the dome, our study is based on an accurate geometrical description of the dome obtained by a geometric survey using a 3D-laser scanner (Figure 7).

Indeed, starting from the cloud of points obtained from the 3D-laser scanner survey, it was possible to obtain analytical expressions of the external profile of the dome, and of the intrados and extrados profiles of the candela using a non-linear polynomial regression of an appropriate degree. For the case study, it was chosen a fourth-degree polynomial approximation.

Then the innovative semi-analytical model described in Section 5 has been implemented in Wolfram Mathematica environment in order to obtain a numerical solution for the present case study.

First, we have considered only the self-weight of the candela $\left(M_{S}^{i}=M_{R}^{i}=0\right)$, in order to obtain the theoretical profile of the candela given by the NFMCT approach in [2]. In this case, by imposing the boundary conditions:

$$
Y(0)=0.49 ; y(0)=0.75 ; y^{\prime}(0)=1 / 3,
$$

the best-fit solution is achieved for an angular width of the dome wedge equal to $\varphi_{0}=41.27^{\circ}$; the obtained theoretical profile of the candela is shown in Figure 8.

The low value of the angle $\varphi$ attests the high efficiency of the dome profile with respect to the self-weight load of the candela. This can be justified by observing that the profile of the considered dome has a very ogival shape, with 

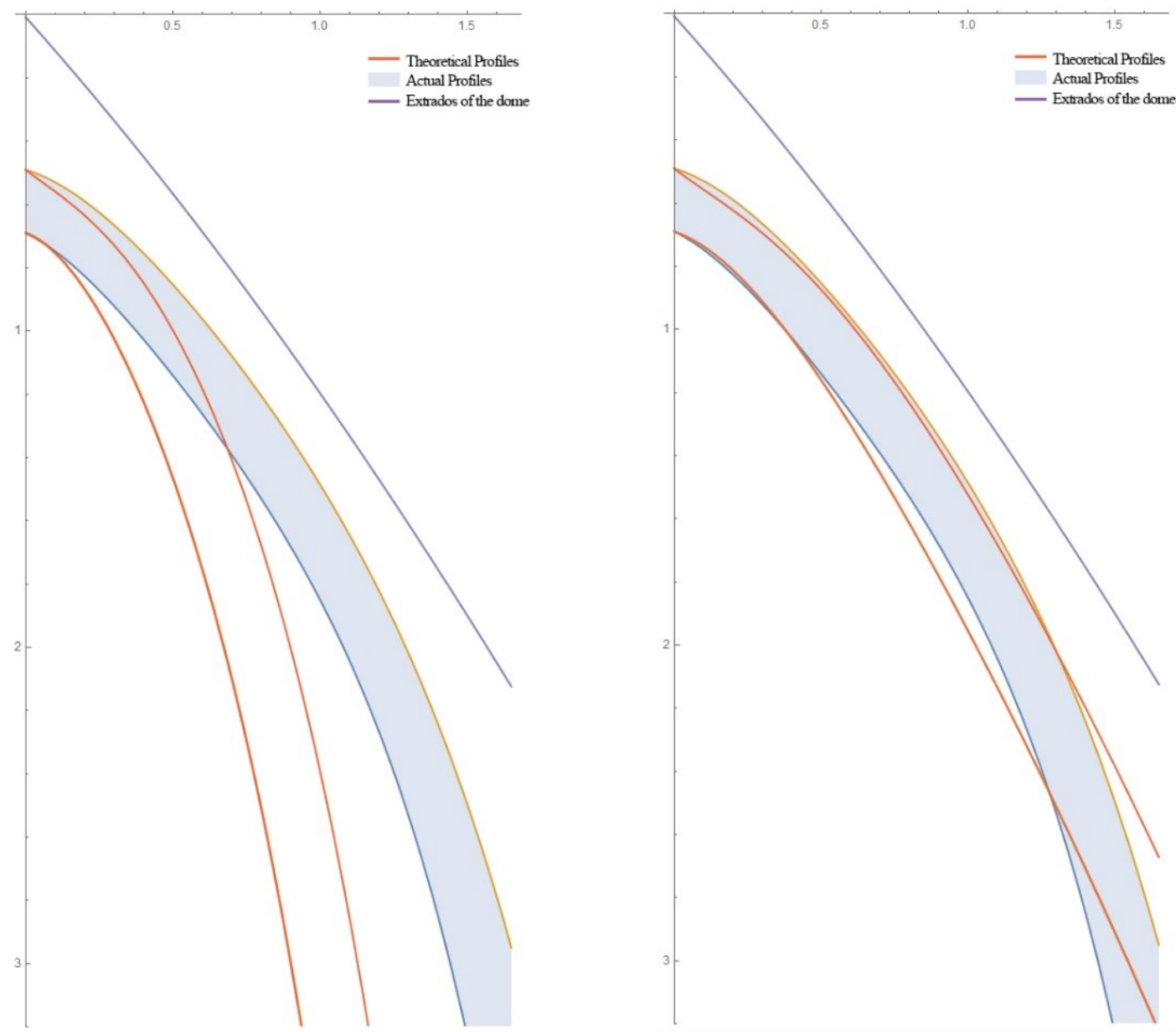

Figure 9: Comparison between actual and theoretical profiles of the candela considering the weight of the infill: (a) solution for the best-fit angle obtained for the only self-weight of the candela $\left(\varphi_{0}=41.27^{\circ}\right)$; (b) solution obtained for $\varphi_{1}=73.37^{\circ}$.

a high ratio between the rise and the span (approximately equal to 1 ).

By considering in the overturning limit analysis also the weight of the infill, that is, by applying the approach here proposed, the solution changes significantly.

Indeed, if we consider the same optimal angle $\varphi_{0}$ obtained in correspondence of the best-fit solution for the self-weight only, the theoretical profiles of the candela shows a significant deviation from the actual profile (Figure 9a).

Now, in order to achieve a best fit solution, we have to consider bigger meridian wedge width angles: the load due to the infill requires more structural capacity resources in order to be carried. In particular, only for $\varphi_{1}=73.37^{\circ}$ it is possible to obtain a theoretical profile of the candela that closely approximate the actual profile of the dome (Figure 9b).

As stated in [13] and in [2], the value of $\varphi$ can be related to the efficiency of the shape of the dome with respect to the considered load condition and then smaller angles $\varphi$ could be related to a greater load-bearing capacity of the dome.

With this in mind, it is interesting to discuss the peculiar behavior of corbelled domes of Trulli with reference to the classical case of masonry vaults as barrel vaults or cross vaults. Indeed, in analogy with the latter, it might be thought that the load of the infill could give a stabilizing contribution to the equilibrium of the whole structure $[22,23]$. But actually, from the results of the above analysis, the load distribution due to the non-structural masses applied at the extrados of the candela has not a stabilizing effect on the equilibrium of the corbelled dome. Rather, the infill load reduces the structural efficiency of the whole structure. Evidence of this is the fact that the best fit solution value of $\varphi$ for the two considered load cases (without or with the load of the infill) is quite different and in particular, when considering the weight of the infill, it is needed a greater value of width angle of the 
meridian wedge $\varphi$ in order the overturning limit equilibrium for the dome to be verified.

\section{Conclusions}

In this paper, we propose a further development of the Corbelling Theory based on the formulation proposed in [2] for the analysis of the limit equilibrium of corbelled domes like that of Alberobello Trulli. In particular, it is wellknown that a corbelled dome of a Trullo is a layered thick shell and that only the thin inner layer (candela) has a structural role.

Starting from the evidence that this layer is subjected to a not negligible load due to the non-structural masses applied at its extrados, it was proposed a further refinement of the semi-analytical NFMCT approach in [2], capable of assessing the structural safety of the dome also considering more complex load conditions.

This approach has been discussed with reference to the paradigmatic case study of a Trullo located in Alberobello.

First, starting from a accurate geometric investigation using the 3D-laser scanner technique, they have been obtained analytical expressions for the actual profiles of the dome using a non-linear polynomial regression.

On this basis, it was applied our innovative approach by considering two load conditions: the first consisting only in the self-weight of the candela, and the second considering also the weight of the infill.

From the analysis of the best-fit solutions, it is possible to better understand some particular aspects of the structural behavior of such kind of corbelled domes. In particular, the non-constant distribution of the non-structural mass applied at the extrados of the dome, differently from the classical case of ordinary masonry vault and dome, does not produce a stabilizing effect for the whole structure. This is shown by the increase of the best-fit solution angle $\varphi$ required to verify the limit overturning equilibrium in presence of the load of the infill with respect to the angle required in presence only of the self-weight load.

Notice that in this analysis the eventual contribution of the infill to the strength of the dome in the spirit of the Limit Analysis framework, was deliberately neglected: this is conservative from the sake of the structural safety.

It can be concluded that the typical conical shape of the outer shell "chiancarelle" layer of the domes of Trulli does not correspond to any static principle but probably it is only a simpler and more economical technical solution to the problem of drainage of the meteoric waters.
Acknowledgement: We acknowledge the major of Alberobello Michele Maria Longo who allowed us the access to the studied Trullo. We are also grateful to Pietro Grimaldi for providing the 3D-laser scanner survey of this Trullo.

\section{References}

[1] E. Benvenuto and M. Corradi, "La statica delle false volte," in Architettura in pietra a secco, Schena Editori, 1987, pp. 93-106.

[2] P. Foti, A. Fraddosio, N. Lepore, and M. D. Piccioni, “On the mechanics of corbelled domes: new analytical and computational approaches," Res. Eng. Struct. Mat, 2016.

[3] N. A. Lazar, J. B. Kadane, F. Chen, W. G. Cavanagh, and C. D. Litton, "Corbelled domes in two and three dimensions: The Treasury of Atreus," Int. Stat. Rev., vol. 72, no. 2, pp. 239-255, 2004.

[4] M. Como, "Statics of Historic Masonry Constructions: An Essay," in Masonry Structures: Between Mechanics and Architecture, 2015.

[5] S. Huerta, Arcos, bóvedas y cúpulas. Geometría y equilibrio en el cálculo tradicional de estructuras de fábrica. Instituto Juan De Herrera, 2004.

[6] D. O’Dwyer, “Funicular analysis of masonry vaults," Comput. Struct., 1999.

[7] E. Ricci, E. Sacco, and M. D. Piccioni, "A method for the analysis of masonry arches," in Structural Analysis of Historical Constructions: Anamnesis, diagnosis, therapy, controls - Proceedings of the 10th International Conference on Structural Analysis of Historical Constructions, SAHC 2016, 2016, pp. 1239-1245.

[8] E. Ricci, A. Fraddosio, M. D. Piccioni, and E. Sacco, "Numerical methods for the lower bound limit analysis of masonry arches," AIMETA 2017 - Proc. 23rd Conf. Ital. Assoc. Theor. Appl. Mech., vol. 2, no. September, 2017.

[9] G. Lacidogna and F. Accornero, "Elastic, plastic, fracture analysis of masonry arches: A multi-span bridge case study," Curved Layer. Struct., 2018.

[10] W. G. Cavanagh and R. R. Laxton, "The structural mechanics of the mycenaean tholos tomb," Annu. Br. Sch. Athens, 1981.

[11] M. T. Como, “L'architettura delle tholoi micenee: analisi degli aspetti compositivi, costruttivi e statici," Università degli Studi "Suor Orsola Benincasa", Napoli, 2005.

[12] M. T. Como, "Analysis of the statics of Mycenaean Tholoi," in Proceedings of the 2th International Congress on Construction History, Cambridge, UK, 2006, pp. 777-790.

[13] L. Rovero and U. Tonietti, "A modified corbelling theory for domes with horizontal layers," Constr. Build. Mater., 2014.

[14] Allen E., Pietre di Puglia: Dolmen, Trulli e Insediamenti Rupestri. Adda Editore, Bari, Italy, 1984.

[15] P. Block, "Thrust network analysis: exploring three-dimensional equilibrium," Massachusetts Institute of Technology, Cambridge, 2009.

[16] P. Foti, A. Fraddosio, N. Lepore, and Mario Daniele Piccioni, "Three-dimensional lower-bound analysis of masonry structures," in Structures and Architecture: Beyond their Limits, CRC Press, 2016, pp. 558-566.

[17] P. Condoleo, A. Taliercio, and L. Binda, "Safety assessment of temple E7 in My So'n, Viet Nam," Struct. Anal. Hist. Constr. Preserv. Saf. significance Proc. Sixth Int. Conf. Struct. Anal. Hist. 
Constr. 2-4 July 2008, Bath, United Kingdom, 2008.

[18] L. Rovero and U. Tonietti, "Structural behaviour of earthen corbelled domes in the Aleppo's region," Mater. Struct. Constr., 2012.

[19] A. Alonso Durá, A. Martínez Boquera, and V. Llopis Pulido, "Analysis and characterization of earthen architecture as a structural material: The corbelled course domes in Syria," in 1st International Conference on Rammed Earth Conservation, RESTAPIA 2012, 2012.

[20] V. Moramarco, I Trulli di Alberobello: Lettura Costruttiva e Indagine Statica. Fasidiluna Editore, Bari, Italy, 2013.

[21] S. Mecca and L. Dipasquale, Earthen domes et habitats: villages of northern Syria. Edizioni ETS, Pisa, Italy, 2009.
[22] J. Heyman, The Stone Skeleton: Structural Engineering of Masonry Structures. Cambridge University Press, Cambridge, UK, 1995.

[23] S. Huerta, "Mechanics of masonry vaults: The equilibrium approach," in 3rd International Seminar in Historical Constructions, Guimarães, Portugal, 2001. 